\title{
Generalized Rough Sets, Entropy, and Image Ambiguity Measures
}

\author{
Debashis Sen, Member, IEEE, and Sankar K. Pal, Fellow, IEEE
}

\begin{abstract}
Quantifying ambiguities in images using fuzzy set theory has been of utmost interest to researchers in the field of image processing. In this paper, we present the use of rough set theory and its certain generalizations for quantifying ambiguities in images and compare it to the use of fuzzy set theory. We propose classes of entropy measures based on rough set theory and its certain generalizations, and perform rigorous theoretical analysis to provide some properties which they satisfy. Grayness and spatial ambiguities in images are then quantified using the proposed entropy measures. We demonstrate the utility and effectiveness of the proposed entropy measures by considering some elementary image processing applications. We also propose a new measure called average image ambiguity in this context.
\end{abstract}

Index Terms-Ambiguity measures, entropy, generalized rough sets, image processing, rough set theory.

\section{INTRODUCTION}

$\mathbf{R}$ EAL-LIFE images are inherently embedded with various ambiguities. For example, imprecision of values at various pixels results in ambiguity, or value gradations cause vague nature of definitions such as region boundaries. Hence, it is natural and appropriate to use techniques that incorporate the ambiguities in order to perform image processing tasks. Fuzzy set theory [1] has been extensively used in order to define various fuzziness measures of an image. The word "fuzziness" has been, in general, related to the ambiguities arising due to the vague definition of region boundaries.

Let us now consider, for example, a $1001 \times 1001$ grayscale image [see Fig. 1(a)] that has sinusoidal gray value gradations in horizontal direction. When an attempt is made to mark the boundary of an arbitrary region in the image, an exact boundary cannot be defined as a consequence of the presence of steadily changing gray values (gray value gradation). This is evident from Fig. 1(b) that shows a portion of the image, where it is known that the pixels in the "white" shaded area uniquely belong to a region. However, the boundary (on the left and right sides) of this region is vague as it can lie anywhere in the gray value gradations present in the portion. Value gradation is a common phenomenon in real-life images, and hence, it is widely accepted that regions in an image have fuzzy boundaries.

The values at various pixels in grayscale images are considered to be imprecise, both in terms of the location and the gray

Manuscript received May 7, 2008; revised August 6, 2008. First published December 2, 2008; current version published January 15, 2009. This paper was recommended by Associate Editor L. Wang.

The authors are with the Center for Soft Computing Research, Indian Statistical Institute, Calcutta 700108, India (e-mail: dsen_t@isical.ac.in; sankar@isical.ac.in).

Digital Object Identifier 10.1109/TSMCB.2008.2005527

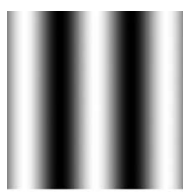

(a)

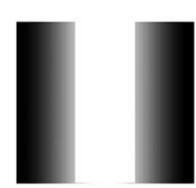

(b)

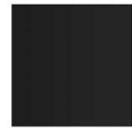

(c)
Fig. 1. Ambiguities in a grayscale image with sinusoidal gray value gradations in horizontal direction. (a) A grayscale image. (b) Fuzzy boundary. (c) Rough resemblance.

level. This means that a gray value at a pixel represents those at its neighboring pixels to certain extents. It also means that a gray value represents nearby gray levels to certain extents. Moreover, pixels in a neighborhood with nearby gray levels have limited discernibility due to the inadequacy of contrast. For example, Fig. 1(c) shows a $6 \times 6$ portion cut from the image in Fig. 1(a). Although this portion contains gray values separated by six gray levels, it appears to be almost homogeneous.

Therefore, from the aforementioned analysis, we find that the ambiguities in a grayscale image are due to the following.

1) Various regions have fuzzy boundaries.

2) Nearby gray levels roughly resemble each other, and values at nearby pixels have rough resemblance.

Ambiguities in a grayscale image are of two types, namely, grayness ambiguity and spatial ambiguity [2]. Grayness ambiguity can be quantified considering the fuzzy boundaries of regions based on global gray value distribution and the rough resemblance between nearby gray levels. On the other hand, spatial ambiguity can be quantified considering the fuzzy boundaries of regions based on organization of gray values at various pixels and the rough resemblance between values at nearby pixels.

The fuzzy set theory of Lofti Zadeh is based on the concept of vague boundaries of sets in the universe of discourse [1]. The rough set theory of Zdzislaw Pawlak, on the other hand, focuses on ambiguity in terms of limited discernibility of sets in the domain of discourse [3]. Therefore, fuzzy sets can be used to represent the ambiguities in images due to the vague definition of region boundaries (fuzzy boundaries), and rough sets can be used to represent the ambiguities due to the indiscernibility between individual or groups of pixels or gray levels (rough resemblance).

Rough set theory, which was initially developed considering crisp equivalence approximation spaces [3], has been generalized by considering fuzzy [4] and tolerance [5] approximation spaces. Furthermore, rough set theory, which was initially developed to approximate crisp sets, has also been generalized to approximate fuzzy sets [4]. 
In this paper, we study the rough set theory and its certain generalizations to quantify ambiguities in images. Here, the generalizations to rough set theory based on the approximation of crisp and fuzzy sets considering crisp equivalence, fuzzy equivalence, crisp tolerance, and fuzzy tolerance approximation spaces in different combinations are studied. All these combinations give rise to different concepts for modeling vagueness, which can be quantified using the roughness measure [3].

We propose classes of entropy measures which use the roughness measures obtained considering the aforementioned various concepts for modeling vagueness. We perform rigorous theoretical analysis of the proposed entropy measures and provide some properties which they satisfy. We then use the proposed entropy measures to quantify ambiguities in images, giving an account of the manner in which the ambiguities are captured. We show that the aforesaid generalizations to rough set theory regarding the approximation of fuzzy sets can be used to quantify ambiguities due to both fuzzy boundaries and rough resemblance.

The utility of the proposed measures in quantifying image ambiguities is demonstrated using some image processing operations like enhancement evaluation, segmentation, and edge detection. A new measure called average image ambiguity (AIA) is also defined in this context. The effectiveness of some of the proposed measures is shown by qualitative and quantitative comparisons of their use in image analysis with that of certain fuzziness measures.

The proposed entropy measures and their properties are presented in Section II, and their utility in quantifying grayness and spatial ambiguities is shown in Section III. Experiments are presented in Section IV to demonstrate the effectiveness of the proposed measures. This paper concludes with Section V.

\section{Entropy Measures With Respect to the DEFinABILITy of A SET OF ElEMENTS}

Defining entropy measures based on rough set theory has been considered by researchers in the past decade. Probably, the first of such work was reported in [6], where a "rough entropy" of a set in a universe has been proposed. This rough entropy measure is defined based on the uncertainty in granulation (obtained using a relation defined over universe [3]) and the definability of the set. Other entropy measures that quantify the uncertainty in crisp or fuzzy granulation alone have been reported in literature [6]-[9]. An entropy measure is presented in [10], which, although not based on rough set theory, quantifies information with the underlying elements having limited discernibility between them.

Incompleteness of knowledge about a universe leads to granulation [3], and hence, a measure of the uncertainty in granulation quantifies this incompleteness of knowledge. Therefore, apart from the "rough entropy" in [6] which quantifies the incompleteness of knowledge about a set in a universe, the other aforesaid entropy measures quantify the incompleteness of knowledge about a universe. The effect of the incompleteness of knowledge about a universe becomes evident only when an attempt is made to define a set in it. Note that the definability of a set in a universe is not always affected by a change in the uncertainty in granulation. This is evident in a few examples given in [6], which we do not repeat here for the sake of brevity. Hence, a measure of the incompleteness of knowledge about a universe with respect to only the definability of a set is required.

The first attempt of formulating an entropy measure with respect to the definability of a set was made by Pal et al. [11], which was used for image segmentation. However, as pointed out in [12], this measure does not satisfy the necessary property that the entropy value is maximum (or optimum) when the uncertainty (in this case, incompleteness of knowledge) is maximum.

In this section, we propose classes of entropy measures, which quantify the incompleteness of knowledge about a universe with respect to the definability of a set of elements (in the universe) holding a particular property (representing a category). An inexactness measure of a set, like the "roughness" measure [3], quantifies the definability of the set. We measure the incompleteness of knowledge about a universe with respect to the definability of a set by considering the roughness measure of the set and also that of its complement in the universe.

\section{A. Roughness of a Set in a Universe}

Let $U$ denote a universe of elements and $X$ be an arbitrary set of elements in $U$ holding a particular property. According to rough set theory [3] and its generalizations, limited discernibility draws elements in $U$ together governed by an indiscernibility relation $R$, and hence, granules of elements are formed in $U$. An indiscernibility relation [3] in a universe refers to the similarities that every element in the universe has with the other elements of the universe. The family of all granules obtained using the relation $R$ is represented as $U / R$. The indiscernibility relation among elements and sets in $U$ results in an inexact definition of $X$. However, the set $X$ can be approximately represented by two exactly definable sets $\underline{R} X$ and $\bar{R} X$ in $U$, which are obtained as

$$
\begin{aligned}
& \underline{R} X=\bigcup\{Y \in U / R: Y \subseteq X\} \\
& \bar{R} X=\bigcup\{Y \in U / R: Y \cap X \neq \emptyset\} .
\end{aligned}
$$

In the aforesaid expressions, $\underline{R} X$ and $\bar{R} X$ are called the $R$-lower approximation and the $R$-upper approximation of $X$, respectively. In essence, the pair of sets $\langle\underline{R} X, \bar{R} X\rangle$ is the representation of any arbitrary set $X \subseteq U$ in the approximation space $\langle U, R\rangle$, where $X$ cannot be defined. As given in [3], an inexactness measure of the set $X$ can be defined as

$$
\rho_{R}(X)=1-\frac{|\underline{R} X|}{|\bar{R} X|}
$$

where $|\underline{R} X|$ and $|\bar{R} X|$ are the cardinalities of the sets $\underline{R} X$ and $\bar{R} X$ in $U$, respectively. The inexactness measure $\rho_{R}(X)$ is called the $R$-roughness measure of $X$, and it takes a value in the interval $[0,1]$.

\section{B. Lower and Upper Approximations of a Set}

The expressions for the lower and upper approximations of the set $X$ depend on the type of relation $R$ and whether $X$ 
TABLE I

DifFERENT NAMES OF $\langle\underline{R} X, \bar{R} X\rangle$ AND $\langle U, R\rangle$

\begin{tabular}{|c|c|c|c|}
\hline$X$ & $R$ & $\langle\underline{R} X, \bar{R} X\rangle$ & $\langle U, R\rangle$ \\
\hline \hline Crisp & $m_{Y}(u) \in\{0,1\}$ (crisp equivalence relation) & rough set of $X$ & crisp equivalence approximation space \\
\hline Fuzzy & $m_{Y}(u) \in\{0,1\}$ (crisp equivalence relation) & rough-fuzzy set of $X$ & crisp equivalence approximation space \\
\hline Crisp & $m_{Y}(u) \in[0,1]$ (fuzzy equivalence relation) & fuzzy rough set of $X$ & fuzzy equivalence approximation space \\
\hline Fuzzy & $m_{Y}(u) \in[0,1]$ (fuzzy equivalence relation) & fuzzy rough-fuzzy set of $X$ & fuzzy equivalence approximation space \\
\hline Crisp & $S_{R}: U \times U \rightarrow\{0,1\}$ (crisp tolerance relation) & tolerance rough set of $X$ & crisp tolerance approximation space \\
\hline Fuzzy & $S_{R}: U \times U \rightarrow\{0,1\}$ (crisp tolerance relation) & tolerance rough-fuzzy set of $X$ & crisp tolerance approximation space \\
\hline Crisp & $S_{R}: U \times U \rightarrow[0,1]$ (fuzzy tolerance relation) & tolerance fuzzy rough set of $X$ & fuzzy tolerance approximation space \\
\hline Fuzzy & $S_{R}: U \times U \rightarrow[0,1]$ (fuzzy tolerance relation) & tolerance fuzzy rough-fuzzy set of $X$ & fuzzy tolerance approximation space \\
\hline
\end{tabular}

is a crisp [1] or a fuzzy [1] set. Here, we shall consider the upper and lower approximations of the set $X$ when $R$ denotes an equivalence, a fuzzy equivalence, a tolerance, or a fuzzy tolerance relation and $X$ is a crisp or a fuzzy set.

When $X$ is a crisp or a fuzzy set and the relation $R$ is a crisp or a fuzzy equivalence relation, the expressions for the lower and upper approximations of the set $X$ are given as

$$
\begin{aligned}
\underline{R} X & =\{(u, \underline{M}(u)) \mid u \in U\} \\
\bar{R} X & =\{(u, \bar{M}(u)) \mid u \in U\}
\end{aligned}
$$

where

$$
\begin{aligned}
& \underline{M}(u)=\sum_{Y \in U / R} m_{Y}(u) \times \inf _{\varphi \in U} \max \left(1-m_{Y}(\varphi), \mu_{X}(\varphi)\right) \\
& \bar{M}(u)=\sum_{Y \in U / R} m_{Y}(u) \times \sup _{\varphi \in U} \min \left(m_{Y}(\varphi), \mu_{X}(\varphi)\right)
\end{aligned}
$$

where the membership function $m_{Y}$ represents the belongingness of every element $(u)$ in the universe $(U)$ to a granule $Y \in U / R$ and it takes values in the interval $[0,1]$ such that $\sum_{Y} m_{Y}(u)=1$, and $\mu_{X}$, which takes values in the interval $[0,1]$, is the membership function associated with $X$. When $X$ is a crisp set, $\mu_{X}$ would take values only from the set $\{0,1\}$. Similarly, when $R$ is a crisp equivalence relation, $m_{Y}$ would take values only from the set $\{0,1\}$. The symbols $\sum$ (sum) and $\times$ (product) in (5) represent specific fuzzy union and intersection operations [1], respectively, which are chosen based on their suitability with respect to the underlying application of measuring ambiguity.

Note that, till now, we have considered the indiscernibility relation $R \subseteq U \times U$ to be an equivalence relation, i.e., $R$ satisfies crisp or fuzzy reflexivity, symmetry, and transitivity properties [1]. However, if $R$ does not satisfy any one of these three properties, the expressions in (4) can no longer be used. We shall consider here the case when the transitivity property is not satisfied. Such a relation $R$ is said to be a tolerance relation, and the space $\langle U, R\rangle$ obtained is referred to as a tolerance approximation space [5]. When $R$ is a tolerance relation, the expressions for the membership values corresponding to the lower and upper approximations [see (5)] of an arbitrary set $X$ in $U$ are given as

$$
\begin{aligned}
& \underline{M}(u)=\inf _{\varphi \in U} \max \left(1-S_{R}(u, \varphi), \mu_{X}(\varphi)\right) \\
& \bar{M}(u)=\sup _{\varphi \in U} \min \left(S_{R}(u, \varphi), \mu_{X}(\varphi)\right)
\end{aligned}
$$

where $S_{R}(u, \varphi)$ is a value representing the tolerance relation $R$ between $u$ and $\varphi$.

The pair of sets $\langle\underline{R} X, \bar{R} X\rangle$ and the approximation space $\langle U, R\rangle$ are referred to differently, depending on whether $X$ is a crisp or a fuzzy set; the relation $R$ is a crisp or a fuzzy equivalence, or a crisp or a fuzzy tolerance relation. The different names are listed in Table I.

\section{Entropy Measures}

As mentioned earlier, the lower and upper approximations of a vaguely definable set $X$ in a universe $U$ can be used in the expression given in (3) in order to get an inexactness measure of the set $X$ called the roughness measure $\rho_{R}(X)$. The vague definition of $X$ in $U$ signifies the incompleteness of knowledge about $U$.

Here, we propose two classes of entropy measures based on the roughness measures of a set and its complement in order to quantify the incompleteness of knowledge about a universe. One of the proposed two classes of entropy measures is obtained by measuring the "gain in information" or, in our case, the "gain in incompleteness" using a logarithmic function as suggested in Shannon's theory. This proposed class of entropy measures for quantifying the incompleteness of knowledge about $U$ with respect to the definability of a set $X \subseteq U$ is given as

$$
H_{R}^{L}(X)=-\frac{1}{2}\left[\varkappa(X)+\varkappa\left(X^{\complement}\right)\right]
$$

where $\varkappa(D)=\rho_{R}(D) \log _{\beta}\left(\rho_{R}(D) / \beta\right)$ for any set $D \subseteq U, \beta$ denotes the base of the logarithmic function used, and $X^{\complement} \subseteq U$ stands for the complement of the set $X$ in the universe. The various entropy measures of this class are obtained by calculating the roughness values $\rho_{R}(X)$ and $\rho_{R}\left(X^{\mathrm{C}}\right)$ considering the different ways of obtaining the lower and upper approximations of the vaguely definable set $X$. Note that the "gain in incompleteness" term is taken as $-\log _{\beta}\left(\rho_{R} / \beta\right)$ in (7), and for $\beta>1$, it takes a value in the interval $[1, \infty]$. The other class of entropy measures proposed is obtained by considering an exponential function [13] to measure the "gain in incompleteness." This second proposed class of entropy measures for quantifying the incompleteness of knowledge about $U$ with respect to the definability of a set $X \subseteq U$ is given as

$$
H_{R}^{E}(X)=\frac{1}{2}\left[\rho_{R}(X) \beta^{\left(\bar{\rho}_{R}(X)\right)}+\rho_{R}\left(X^{\mathrm{C}}\right) \beta^{\left(\bar{\rho}_{R}\left(X^{\mathrm{C}}\right)\right)}\right]
$$


where $\bar{\rho}_{R}(D)=1-\rho_{R}(D)$ for any set $D \subseteq U$ and $\beta$ denotes the base of the exponential function used. Pal and Pal [13] had considered only the case when $\beta$ equaled $e$. Similar to the class of entropy measures $H_{R}^{L}$, the various entropy measures of this class are obtained by using the different ways of obtaining the lower and upper approximations of $X$ in order to calculate $\rho_{R}(X)$ and $\rho_{R}\left(X^{\mathrm{C}}\right)$. The "gain in incompleteness" term is taken as $\beta^{\left(1-\rho_{R}\right)}$ in (8), and for $\beta>1$, it takes a value in the finite interval $[1, \beta]$. Note that an analysis on the appropriate values that $\beta$ in $H_{R}^{L}$ and $H_{R}^{E}$ can take will be given later in Section II-E-1.

We shall name a proposed entropy measure using attributes that represent the class (logarithmic or exponential) it belongs to and the type of the pair of sets $\langle\underline{R} X, \bar{R} X\rangle$ considered. For example, if $\langle\underline{R} X, \bar{R} X\rangle$ represents a tolerance rough-fuzzy set and the expression of the proposed entropy in (8) is considered, then we call such an entropy as the exponential tolerance rough-fuzzy entropy. Some other examples of names for the proposed entropy measures are the logarithmic rough entropy, the exponential fuzzy rough entropy, and the logarithmic tolerance fuzzy rough-fuzzy entropy.

\section{Relation Between $\rho_{R}(X)$ and $\rho_{R}\left(X^{\complement}\right)$}

Let us first consider a brief discussion on fuzzy-set-theorybased uncertainty measures. Assume that a set FS is fuzzy in nature, and it is associated with a membership function $\mu_{\mathrm{FS}}$. As mentioned in [14], most of the appropriate fuzzy-set-theorybased uncertainty measures can be grouped into two classes, namely, the multiplicative class and the additive class. It should be noted from [14] that the measures belonging to these classes are functions of $\mu_{\mathrm{FS}}$ and $\mu_{\mathrm{FS}} \mathrm{C}$, where $\mu_{\mathrm{FS}}=1-\mu_{\mathrm{FS}^{\mathrm{C}}}$.

Now, as mentioned in [14], the existence of an exact relation between $\mu_{\mathrm{FS}}$ and $\mu_{\mathrm{FS}}$ c suggests that they "theoretically" convey the same. However, sometimes, such unnecessary terms should be retained, as dropping them would cause the corresponding measures to fail certain important properties.

We shall now analyze the relation between $\rho_{R}(X)$ and $\rho_{R}\left(X^{\mathrm{C}}\right)$ and show that there exist no unnecessary terms in the classes of entropy measures [see (7) and (8)] proposed using rough set theory and its certain generalizations. Now, as $\rho_{R}(X)$ takes a value in the interval $[0,1]$, let us consider

$$
\rho_{R}(X)=\frac{1}{C}, \quad 1 \leq C \leq \infty .
$$

Let us now find the range of values that $\rho_{R}\left(X^{\complement}\right)$ can take when the value of $\rho_{R}(X)$ is given. Let the total number of elements in the universe $U$ under consideration be $n$. As we have $X \cup$ $X^{\complement}=U$, it can be easily deduced that $\bar{R} X \cup \underline{R} X^{\complement}=U$ and $\underline{R} X \cup \bar{R} X^{\complement}=U$. Therefore, from (3), we get

$$
\begin{aligned}
& \rho_{R}(X)=1-\frac{|\underline{R} X|}{|\bar{R} X|} \\
& \rho_{R}\left(X^{\complement}\right)=1-\frac{\left|\underline{R} X^{\complement}\right|}{\left|\bar{R} X^{\complement}\right|}=1-\frac{n-|\bar{R} X|}{n-|\underline{R} X|} .
\end{aligned}
$$

From (9), (10) and (11), we deduce that

$$
\rho_{R}\left(X^{\complement}\right)=\rho_{R}(X) \frac{|\bar{R} X|}{n-|\underline{R} X|}=\frac{1}{C}\left(\frac{|\bar{R} X|}{n-|\underline{R} X|}\right) .
$$

We shall now separately consider three cases of (12), where we have $1<C<\infty, C=1$, and $C=\infty$.

When we have $1<C<\infty$, we get the relation $(|\underline{R} X|)$ $|\bar{R} X|)=(C-1) / C$ from (9). Using this relation in (12), we obtain

$$
\rho_{R}\left(X^{\complement}\right)=\frac{1}{C}\left(\frac{|\underline{R} X|\left(\frac{C}{C-1}\right)}{n-|\underline{R} X|}\right) .
$$

After some algebraic manipulations, we deduce that

$$
\rho_{R}\left(X^{\mathrm{C}}\right)=\frac{1}{C-1}\left(\frac{1}{\frac{n}{|\underline{R} X|}-1}\right) .
$$

Note that when $1<C<\infty, \rho_{R}(X)$ takes a value in the interval $(0,1)$. Therefore, in this case, the value of $|\bar{R} X|$ could range from a positive infinitesimal quantity, for example, $\epsilon$, to a maximum value of $n$. Hence, we have

$$
\epsilon \frac{C-1}{C} \leq|\underline{R} X| \leq n \frac{C-1}{C} .
$$

Using (15) in (14), we get

$$
\frac{\epsilon}{n C-\epsilon(C-1)} \leq \rho_{R}\left(X^{\complement}\right) \leq 1 .
$$

As $1<C<\infty, \epsilon \ll 1$, and, usually, $n \gg 1$, we may write (16) as

$$
0<\rho_{R}\left(X^{\complement}\right) \leq 1 .
$$

Thus, we may conclude that, for a given nonzero and nonunity value of $\rho_{R}(X), \rho_{R}\left(X^{\complement}\right)$ may take any value in the interval $(0,1]$.

When $C=1$ or $\rho_{R}(X)$ takes a unity value, $|\underline{R} X|=0$, and the value of $|\bar{R} X|$ could range from $\epsilon$ to a maximum value of $n$. Therefore, it is easily evident from (12) that $\rho_{R}\left(X^{\mathrm{C}}\right)$ may take any value in the interval $(0,1]$ when $\rho_{R}(X)=1$.

Let us now consider the case when $C=\infty$ or $\rho_{R}(X)=0$. In such a case, the value of $|\bar{R} X|$ could range from zero to a maximum value of $n$, and $|\underline{R} X|=|\bar{R} X|$. As evident from (12), when $C=\infty$, irrespective of any other term, we get $\rho_{R}\left(X^{\complement}\right)=0$. This is obvious, as an exactly definable set $X$ should imply an exactly definable set $X^{\complement}$.

Therefore, we find that the relation between $\rho_{R}(X)$ and $\rho_{R}\left(X^{\mathrm{C}}\right)$ is such that if one of them is considered to take a nonzero value (i.e., the underlying set is vaguely definable or inexact), the value of the other, which would also be a nonzero quantity, cannot be uniquely specified. Therefore, there exist no unnecessary terms in the proposed classes of entropy measures given in (7) and (8). However, from (10) and (11), it is easily evident that $\rho_{R}(X)$ and $\rho_{R}\left(X^{\mathrm{C}}\right)$ are positively correlated.

\section{E. Properties of the Proposed Classes of Entropy Measures}

In this section, till now, we have proposed two classes of entropy measures, and we have shown that the expressions for 
the proposed entropy measures do not have any unnecessary terms. However, the base parameters $\beta$ 's [see (7) and (8)] of the two classes of entropy measures incur certain restrictions, so that the proposed entropies satisfy some important properties. In this section, we shall discuss the restrictions regarding the base parameters and then provide few properties of the proposed entropies.

1) Range of Values for the Base $\beta$ : The proposed classes of entropy measures $H_{R}^{L}$ and $H_{R}^{E}$ given in (7) and (8), respectively, must be consistent with the fact that maximum information (entropy) is available when the uncertainty is maximum and the entropy is zero when there is no uncertainty. Note that, in our case, maximum uncertainty represents maximum possible incompleteness of knowledge about the universe. Therefore, maximum uncertainty occurs when both the roughness values used in $H_{R}^{L}$ and $H_{R}^{E}$ equal unity, and uncertainty is zero when both of them are zero. It can be easily shown that in order to satisfy the aforesaid condition, the base $\beta$ in $H_{R}^{L}$ must take a finite value greater than or equal to $e(\approx 2.7183)$, and the base $\beta$ in $H_{R}^{E}$ must take a value in the interval $(1, e]$. When $\beta \geq e$ in $H_{R}^{L}$ and $1<\beta \leq e$ in $H_{R}^{E}$, the values taken by both $H_{R}^{L}$ and $H_{R}^{E}$ lie in the range $[0,1]$. Note that for an appropriate $\beta$ value, the proposed entropy measures attain the minimum value of zero only when $\rho_{R}(X)=\rho_{R}\left(X^{\mathrm{C}}\right)=0$ and the maximum value of unity only when $\rho_{R}(X)=\rho_{R}\left(X^{\mathrm{C}}\right)=1$.

2) Properties: Here, we present few properties of the proposed logarithmic and exponential classes of entropy measures expressing $H_{R}^{L}$ and $H_{R}^{E}$ as functions of two parameters representing roughness measures. We may rewrite the expressions given in (7) and (8) in parametric form as follows, respectively:

$$
\begin{aligned}
& H_{R}^{L}(A, B)=-\frac{1}{2}\left[A \log _{\beta}\left(\frac{A}{\beta}\right)+B \log _{\beta}\left(\frac{B}{\beta}\right)\right] \\
& H_{R}^{E}(A, B)=\frac{1}{2}\left[A \beta^{(1-A)}+B \beta^{(1-B)}\right]
\end{aligned}
$$

where the parameters $A(\in[0,1])$ and $B(\in[0,1])$ represent the roughness values $\rho_{R}(X)$ and $\rho_{R}\left(X^{\complement}\right)$, respectively. Considering the convention $0 \log _{\beta} 0=0$, let us now discuss the following properties of $H_{R}^{L}(A, B)$ and $H_{R}^{E}(A, B)$ along the lines of [15].

1) Nonnegativity: We have $H_{R}^{L}(A, B) \geq 0$ and $H_{R}^{E}(A$, $B) \geq 0$ with equality in both the cases if and only if $A=0$ and $B=0$.

2) Continuity: Both $H_{R}^{E}(A, B)$ and $H_{R}^{L}(A, B)$ are continuous functions of $A$ and $B$, where $A, B \in[0,1]$.

3) Sharpness: Both $H_{R}^{L}(A, B)$ and $H_{R}^{E}(A, B)$ equal zero if and only if the roughness values $A$ and $B$ equal zero, i.e., $A$ and $B$ are "sharp."

4) Maximality and Normality: Both $H_{R}^{L}(A, B)$ and $H_{R}^{E}(A$, $B$ ) attain their maximum value of unity if and only if the roughness values $A$ and $B$ are unity. That is, we have $H_{R}^{L}(A, B) \leq H_{R}^{L}(1,1)=1$ and $H_{R}^{E}(A, B) \leq$ $H_{R}^{E}(1,1)=1$, where $A, B \in[0,1]$.

5) Resolution: We have $H_{R}^{L}\left(A^{*}, B^{*}\right) \leq H_{R}^{L}(A, B)$ and $H_{R}^{E}\left(A^{*}, B^{*}\right) \leq H_{R}^{E}(A, B)$, where $A^{*}$ and $B^{*}$ are the

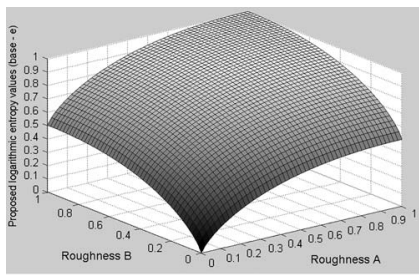

(a)

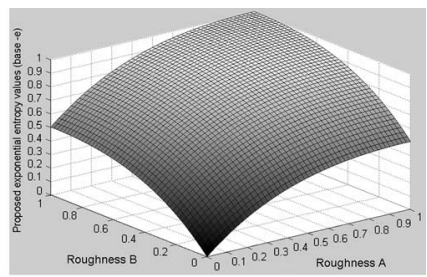

(b)
Fig. 2. Plots of the proposed classes of entropy measures for various roughness values $A$ and $B$. (a) Logarithmic. (b) Exponential.

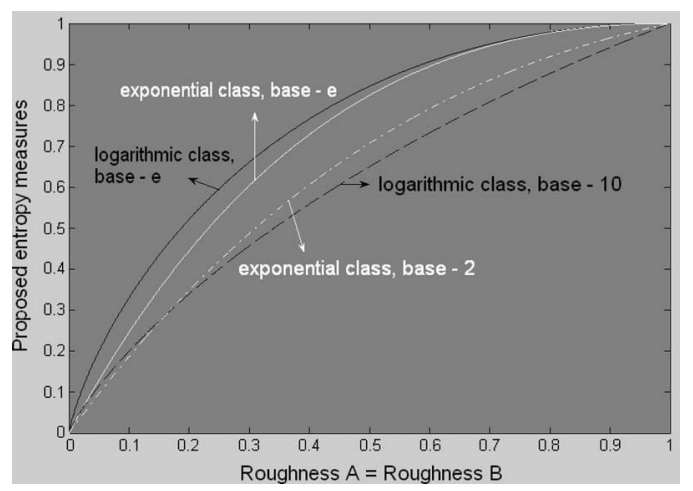

Fig. 3. Proposed entropy measures for a few different $\beta$ values, when $A=B$.

sharpened versions of $A$ and $B$, respectively, i.e., $A^{*} \leq A$ and $B^{*} \leq B$.

6) Symmetry: Both $H_{R}^{L}(A, B)$ and $H_{R}^{E}(A, B)$ are symmetric about the line $A=B$.

7) Monotonicity: Both $H_{R}^{L}(A, B)$ and $H_{R}^{E}(A, B)$ are monotonically nondecreasing functions of $A$ and $B$.

8) Concavity: Both $H_{R}^{L}(A, B)$ and $H_{R}^{E}(A, B)$ are concave functions of $A$ and $B$.

The plots of the proposed classes of entropies $H_{R}^{L}$ and $H_{R}^{E}$ as functions of $A$ and $B$ are shown in Figs. 2 and 3, respectively. In Fig. 2, the values of $H_{R}^{L}$ and $H_{R}^{E}$ are shown for all possible values of the roughness measures $A$ and $B$ considering $\beta=e$. Fig. 3 shows the plots of the proposed entropies for different values of the base $\beta$, when $A=B$.

\section{Measuring Ambiguities In Images}

Ambiguities in grayscale images are due to fuzzy boundaries between regions, and rough resemblance between nearby gray levels and between values at nearby pixels (see Section I). In this section, we shall use the entropy measures proposed in the previous section in order to quantify ambiguities in a grayscale image. As we shall see later, the entropy measures based on the generalization of rough set theory regarding the approximation of fuzzy sets (i.e., when the set $X$ considered in the previous section is fuzzy) can be used to quantify ambiguities due to both fuzzy boundaries and rough resemblance, whereas the entropy measures based on the generalization of rough set theory regarding the approximation of crisp sets (i.e., when the set $X$ considered in the previous section is crisp) can be used to quantify ambiguities only due to rough resemblance. 
As mentioned in Section I, ambiguities in a grayscale image are of two types, namely, grayness and spatial ambiguities. Grayness ambiguity measure can be obtained by considering the fuzzy boundaries of regions based on global gray value distribution and the rough resemblance between nearby gray levels. In this case, the image should be considered as an array of gray values, and the measure of consequence of the incompleteness of knowledge about the universe of gray levels in the array would quantify the ambiguities. Spatial ambiguity measure can be obtained by considering the fuzzy boundaries of regions based on organization of gray values at various pixels and the rough resemblance between values at nearby pixels. In this case, the image should be considered as a universe of pixels, and the measure of the incompleteness of knowledge about the universe of pixels would quantify the ambiguities. In the aforesaid discussion, the measure of the incompleteness of knowledge about a universe with respect to the definability of a set should be used, as the set would be employed to capture the vagueness in region boundaries.

Note that although the discussion in this section will be on grayscale images, it is also applicable to images obtained by carrying out operations on grayscale images, for example, images of edge strengths.

\section{A. Grayness Ambiguity Measure: Ambiguities in an Image Represented as an Array of Gray Values}

Let $G$ be the universe of gray levels and $\Upsilon_{T}$ be a set in $G$, i.e., $\Upsilon_{T} \subseteq G$, whose elements hold a particular property to extents given by a membership function $\mu_{T}$ defined on $G$. Let us now take up the problem of quantifying ambiguities in an image $I$ considering it as an array of gray values. Let $O_{I}$ be the graylevel histogram of the image $I$. The fuzzy boundaries and rough resemblance in $I$ causing the ambiguities are related to the incompleteness of knowledge about $G$, which can be quantified using the proposed entropy measures in Section II-C.

We shall consider $\Upsilon_{T}$ such that it represents the category "dark areas" in the image $I$, and the associated property "darkness" given by the membership function $\mu_{T}$ shall be modeled as

$$
\mu_{T}(l)= \begin{cases}1, & l \leq T-\Delta \\ 1-2\left[\frac{(l-(T-\Delta))}{2 \Delta}\right]^{2}, & T-\Delta \leq l \leq T \\ 2\left[\frac{(l-(T+\Delta))}{2 \Delta}\right]^{2}, & T \leq l \leq T+\Delta \\ 0, & l \geq T+\Delta\end{cases}
$$

where $l \in G$ and $T$ and $\Delta$ are called the crossover point and the bandwidth, respectively. We shall consider that $\Delta$ is a constant and that different definitions of the property "darkness" can be obtained by changing the value of $T$, where $T \in G$.

In order to quantify the ambiguities in the image $I$ using the proposed classes of entropy measures, we consider the following sets:

$$
\begin{aligned}
& \Upsilon_{T}=\left\{\left(l, \mu_{T}(l)\right) \mid l \in G\right\} \\
& \Upsilon_{T}^{\complement}=\left\{\left(l, 1-\mu_{T}(l)\right) \mid l \in G\right\} .
\end{aligned}
$$

The fuzzy sets $\Upsilon_{T}$ and $\Upsilon_{T}^{\complement}$ previously considered capture the fuzzy boundary aspect of the ambiguities. Furthermore, we consider limited discernibility among the elements in $G$ that results in vague definitions of the fuzzy sets $\Upsilon_{T}$ and $\Upsilon_{T}^{C}$, and hence, the rough resemblance aspect of the ambiguities is also captured.

Granules, with crisp or fuzzy boundaries, are induced in $G$ as its elements are drawn together due to the presence of limited discernibility (or indiscernibility relation) among them, and this process is referred to as the gray-level granulation. We assume that the indiscernibility relation is uniform in $G$, and hence, the granules formed have a constant support cardinality (size) $\omega$. Now, using (4)-(6), we get general expressions for the different lower and upper approximations of $\Upsilon_{T}$ and $\Upsilon_{T}^{\complement}$ obtained considering the different indiscernibility relations discussed in Section II-B as follows:

$$
\begin{aligned}
& \underline{\Upsilon_{T}}=\left\{\left(l, M_{\underline{\Upsilon_{T}}}(l)\right)\right\}, \overline{\Upsilon_{T}}=\left\{\left(l, M_{\overline{\Upsilon_{T}}}(l)\right)\right\} \\
& \underline{\Upsilon_{T}^{\complement}}=\left\{\left(l, M_{\underline{\Upsilon_{T}^{\complement}}}(l)\right)\right\}, \overline{\Upsilon_{T}^{\complement}}=\left\{\left(l, M_{\overline{\Upsilon_{T}^{\complement}}}(l)\right)\right\}
\end{aligned}
$$

where $l \in G$. We have

$$
\begin{aligned}
& M_{\underline{\Upsilon_{T}}}(l)=\sum_{i=1}^{\gamma} m_{\digamma_{i}^{\omega}}(l) \times \inf _{\varphi \in G} \max \left(\bar{m}_{\digamma_{i}^{\omega}}(\varphi), \mu_{T}(\varphi)\right) \\
& M_{\overline{\Upsilon_{T}}}(l)=\sum_{i=1}^{\gamma} m_{\digamma_{i}^{\omega}}(l) \times \sup _{\varphi \in G} \min \left(m_{\digamma_{i}^{\omega}}(\varphi), \mu_{T}(\varphi)\right) \\
& M_{\underline{\Upsilon_{T}^{c}}}(l)=\sum_{i=1}^{\gamma} m_{\digamma_{i}^{\omega}}(l) \times \inf _{\varphi \in G} \max \left(\bar{m}_{\digamma_{i}^{\omega}}(\varphi), \bar{\mu}_{T}(\varphi)\right) \\
& M_{\overline{\Upsilon_{T}^{0}}}(l)=\sum_{i=1}^{\gamma} m_{\digamma_{i}^{\omega}}(l) \times \sup _{\varphi \in G} \min \left(m_{\digamma_{i}^{\omega}}(\varphi), \bar{\mu}_{T}(\varphi)\right)
\end{aligned}
$$

when equivalence indiscernibility relation is considered, with $\bar{m}_{\digamma_{i}^{\omega}}(\varphi)=1-m_{\digamma_{i}^{\omega}}(\varphi)$ and $\bar{\mu}_{T}(\varphi)=1-\mu_{T}(\varphi)$, and we have

$$
\begin{aligned}
& M_{\underline{\Upsilon_{T}}}(l)=\inf _{\varphi \in G} \max \left(\bar{S}_{\omega}(l, \varphi), \mu_{T}(\varphi)\right) \\
& M_{\overline{\Upsilon_{T}}}(l)=\sup _{\varphi \in G} \min \left(S_{\omega}(l, \varphi), \mu_{T}(\varphi)\right) \\
& M_{\underline{\Upsilon_{T}^{\mathrm{c}}}}(l)=\inf _{\varphi \in G} \max \left(\bar{S}_{\omega}(l, \varphi), \bar{\mu}_{T}(\varphi)\right) \\
& M_{\overline{\Upsilon_{T}^{\mathrm{c}}}}(l)=\sup _{\varphi \in G} \min \left(S_{\omega}(l, \varphi), \bar{\mu}_{T}(\varphi)\right)
\end{aligned}
$$

when tolerance indiscernibility relation is considered, with $\bar{S}_{\omega}(l, \varphi)=1-S_{\omega}(l, \varphi)$. In (23), $\gamma$ denotes the number of granules formed in the universe $G$, and $m_{\digamma_{i}^{\omega}}(l)$ gives the membership grade of $l$ in the $i$ th granule $\digamma_{i}^{\omega}$. These membership grades may be calculated using any concave, symmetric, and normal membership function (with support cardinality $\omega$ ), such as the one having a triangular, trapezoidal, or bell (for example, the $\pi$ function) shape. Note that the sum of these membership 


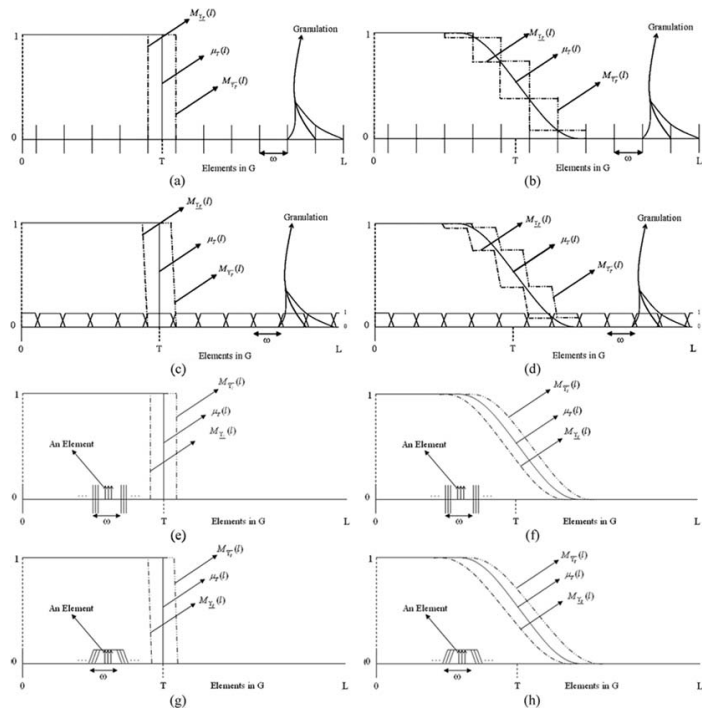

Fig. 4. Different forms that the lower and upper approximations of $\Upsilon_{T}$ can take when used to get the grayness ambiguity measure. (a) Crisp $\Upsilon_{T}$ and Crisp $\digamma_{i}^{\omega}$. (b) Fuzzy $\Upsilon_{T}$ and Crisp $\digamma_{i}^{\omega}$. (c) Crisp $\Upsilon_{T}$ and Fuzzy $\digamma_{i}^{\omega}$. (d) Fuzzy $\Upsilon_{T}$ and Fuzzy $\digamma_{i}^{\omega}$. (e) Crisp $\Upsilon_{T}$ and $S_{\omega}: G \times G \rightarrow\{0,1\}$. (f) Fuzzy $\Upsilon_{T}$ and $S_{\omega}: G \times G \rightarrow\{0,1\}$. (g) Crisp $\Upsilon_{T}$ and $S_{\omega}: G \times G \rightarrow$ $[0,1]$. (h) Fuzzy $\Upsilon_{T}$ and $S_{\omega}: G \times G \rightarrow[0,1]$.

grades over all the granules must be unity for a particular value of $l$. In (24), $S_{\omega}: G \times G \rightarrow[0,1]$, which can be any concave and symmetric function, gives the relation between any two gray levels in $G$. The value of $S_{\omega}(l, \varphi)$ is zero when the difference between $l$ and $\varphi$ is greater than $\omega$, and $S_{\omega}(l, \varphi)$ equals unity when $l$ equals $\varphi$.

The lower and upper approximations of the sets $\Upsilon_{T}$ and $\Upsilon_{T}^{C}$ take different forms, depending on the nature of rough resemblance considered and whether the need is to capture ambiguities due to both fuzzy boundaries and rough resemblance or only those due to rough resemblance. The nature of rough resemblance may be considered such that an equivalence relation between gray levels induces granules having crisp (crisp $\digamma_{i}^{\omega}$ ) or fuzzy (fuzzy $\digamma_{i}^{\omega}$ ) boundaries, or there exists a tolerance relation between gray levels that may be crisp $\left(S_{\omega}: G \times G \rightarrow\right.$ $\{0,1\})$ or fuzzy $\left(S_{\omega}: G \times G \rightarrow[0,1]\right)$. When the sets $\Upsilon_{T}$ and $\Upsilon_{T}^{C}$ considered are fuzzy sets, ambiguities due to both fuzzy boundaries and rough resemblance would be captured, whereas when the sets $\Upsilon_{T}$ and $\Upsilon_{T}^{\complement}$ considered are crisp sets, only the ambiguities due to rough resemblance would be captured. The different forms of the lower and upper approximations of $\Upsilon_{T}$ are shown graphically in Fig. 4.

We shall now quantify the ambiguities in the image $I$ by measuring the consequence of the incompleteness of knowledge about the universe of gray levels $G$ in $I$. This measurement is done by calculating the following values:

$$
\begin{gathered}
\varrho_{\omega}\left(\Upsilon_{T}\right)=1-\frac{\sum_{l \in G} M_{\Upsilon_{T}}(l) O_{I}(l)}{\sum_{l \in G} M_{\overline{\Upsilon_{T}}}(l) O_{I}(l)} \\
\varrho_{\omega}\left(\Upsilon_{T}^{C}\right)=1-\frac{\sum_{l \in G} M_{\Upsilon_{T}^{c}}(l) O_{I}(l)}{\sum_{l \in G} M} \overline{\overline{\Upsilon_{T}^{c}}(l) O_{I}(l)} .
\end{gathered}
$$

The ambiguity measure $\Lambda$ of $I$ is obtained as a function of $T$, which characterizes the underlying set $\Upsilon_{T}$, as follows:

$$
\Lambda_{\omega}^{L}(T)=-\frac{1}{2}\left[\varkappa\left(\Upsilon_{T}\right)+\varkappa\left(\Upsilon_{T}^{\complement}\right)\right]
$$

where $\varkappa(D)=\varrho_{\omega}(D) \log _{\beta}\left(\varrho_{\omega}(D) / \beta\right)$ for any set $D \subseteq G$. Note that the aforementioned expression is obtained by using $\varrho_{\omega}\left(\Upsilon_{T}\right)$ and $\varrho_{\omega}\left(\Upsilon_{T}^{\complement}\right)$ in the proposed logarithmic class of entropy functions given in (8), instead of roughness measures. When the proposed exponential class of entropy functions is used, we get

$$
\Lambda_{\omega}^{E}(T)=\frac{\varrho_{\omega}\left(\Upsilon_{T}\right) \beta^{\left(\bar{\varrho}_{\omega}\left(\Upsilon_{T}\right)\right)}+\varrho_{\omega}\left(\Upsilon_{T}^{\complement}\right) \beta^{\left(\varrho_{\omega}\left(\Upsilon_{T}^{\complement}\right)\right)}}{2}
$$

where $\varrho_{\omega}(D)=1-\varrho_{\omega}(D)$ for any set $D \subseteq G$. It should be noted that the values $\varrho_{\omega}\left(\Upsilon_{T}\right)$ and $\varrho_{\omega}\left(\Upsilon_{T}^{\complement}\right)$ in (25) are obtained by considering "weighted cardinality" measures instead of cardinality measures, which are used for calculating roughness values [see (3)]. The weights considered are the number of occurrences of gray values given by the gray-level histogram $O_{I}$ of the image $I$.

The ambiguity measures obtained using (26) and (27) are referred to as the grayness ambiguity measures, and they lie in the range $[0,1]$, where a larger value means higher ambiguity.

\section{B. Spatial Ambiguity Measure: Ambiguities in an Image Represented as a Universe of Pixels}

Let us now take up the problem of quantifying the ambiguities in an image $I$ considering it as a universe of pixels (associated with gray values). Let $P$ be the universe of pixels and $\Upsilon_{T}$ be a set in $P$, i.e., $\Upsilon_{T} \subseteq P$, whose elements which are associated with gray values hold a particular property to extents given by the membership function $\mu_{T}$ [see (20)] defined on $G$. Now, the fuzzy boundaries and rough resemblance in $I$ causing the ambiguities are related to the incompleteness of knowledge about $P$, which can be quantified using the proposed classes of entropy measures in Section II-C. In order to quantify the ambiguities in the image $I$ using the proposed classes of entropy measures, we consider the following sets:

$$
\begin{aligned}
& \Upsilon_{T}=\left\{\left(\left\langle p_{1}, p_{2}\right\rangle, \mu_{T}\left(l_{\left\langle p_{1}, p_{2}\right\rangle}\right)\right\}\right. \\
& \Upsilon_{T}^{C}=\left\{\left(\left\langle p_{1}, p_{2}\right\rangle, 1-\mu_{T}\left(l_{\left\langle p_{1}, p_{2}\right\rangle}\right)\right\}\right.
\end{aligned}
$$

where $\left\langle p_{1}, p_{2}\right\rangle \in P$ and $l_{\left\langle p_{1}, p_{2}\right\rangle}$ is the gray value at the pixel $\left\langle p_{1}, p_{2}\right\rangle$. Hereafter in this paper, we shall use $p_{12} \equiv\left\langle p_{1}, p_{2}\right\rangle$. The fuzzy sets $\Upsilon_{T}$ and $\Upsilon_{T}^{\complement}$ previously considered capture the fuzzy boundary aspect of the ambiguities. Limited discernibility is considered among the elements in $P$ that results in vague definitions of the fuzzy sets $\Upsilon_{T}$ and $\Upsilon_{T}^{C}$ in (28), and hence the rough resemblance aspect of ambiguities is also captured.

Granules, with crisp or fuzzy boundaries, are induced in $P$ as its elements are drawn together due to the presence of an indiscernibility relation among them, and this process is referred to as the spatial granulation. The indiscernibility relation is assumed uniform in $P$, and hence, the granules formed have a constant support cardinality (size) $\omega_{1} \times \omega_{2}$ denoted as 
$\left\langle\omega_{1}, \omega_{2}\right\rangle$. Now, using (4)-(6), we get general expressions for the differentde lower and upper approximations of $\Upsilon_{T}$ and $\Upsilon_{T}^{\complement}$ given in (28) as follows:

$$
\begin{array}{ll}
\underline{\Upsilon_{T}}=\left\{\left(p_{12}, M_{\Upsilon_{T}}\left(p_{12}\right)\right)\right\} & \overline{\Upsilon_{T}}=\left\{\left(p_{12}, M_{\overline{\Upsilon_{T}}}\left(p_{12}\right)\right)\right\} \\
\underline{\Upsilon_{T}^{\complement}}=\left\{\left(p_{12}, M_{\underline{\Upsilon_{T}^{\complement}}}\left(p_{12}\right)\right)\right\} & \overline{\Upsilon_{T}^{\complement}}=\left\{\left(p_{12}, M_{\overline{\Upsilon_{T}^{\complement}}}\left(p_{12}\right)\right)\right\}
\end{array}
$$

where $p_{12} \in P$. We have $M_{\underline{\Upsilon_{T}}}\left(p_{12}\right), M_{\overline{\Upsilon_{T}}}\left(p_{12}\right), M_{\underline{\Upsilon_{T}^{C}}}\left(p_{12}\right)$, and $M_{\overline{\Upsilon_{T}^{c}}}\left(p_{12}\right)$, respectively, as

$$
\begin{aligned}
& \sum_{i=1}^{\gamma} m_{\digamma_{i}^{\omega_{12}}}\left(p_{12}\right) \inf _{\varphi_{12} \in P} \max \left(\bar{m}_{\digamma_{i}^{\omega_{12}}}\left(\varphi_{12}\right), \mu_{T}\left(l_{\varphi_{12}}\right)\right) \\
& \sum_{i=1}^{\gamma} m_{\digamma_{i}^{\omega_{12}}}\left(p_{12}\right) \sup _{\varphi_{12} \in P} \min \left(m_{\digamma_{i}^{\omega_{12}}}\left(\varphi_{12}\right), \mu_{T}\left(l_{\varphi_{12}}\right)\right) \\
& \sum_{i=1}^{\gamma} m_{\digamma_{i}^{\omega_{12}}}\left(p_{12}\right) \inf _{\varphi_{12} \in P} \max \left(\bar{m}_{\digamma_{i}^{\omega_{12}}}\left(\varphi_{12}\right), \bar{\mu}_{T}\left(l_{\varphi_{12}}\right)\right) \\
& \sum_{i=1}^{\gamma} m_{\digamma_{i}^{\omega_{12}}}\left(p_{12}\right) \sup _{\varphi_{12} \in P} \min \left(m_{\digamma_{i}^{\omega_{12}}}\left(\varphi_{12}\right), \bar{\mu}_{T}\left(l_{\varphi_{12}}\right)\right)
\end{aligned}
$$

when equivalence indiscernibility relation is considered, with $\bar{m}_{\digamma_{i}^{\omega_{12}}}\left(\varphi_{12}\right)=1-m_{\digamma_{i}^{\omega_{12}}}\left(\varphi_{12}\right)$ and $\bar{\mu}_{T}\left(l_{\varphi_{12}}\right)=1-\mu_{T}\left(l_{\varphi_{12}}\right)$, and we have

$$
\begin{aligned}
& M_{\underline{\Upsilon_{T}}}\left(p_{12}\right)=\inf _{\varphi_{12} \in P} \max \left(\bar{S}_{\omega_{12}}\left(p_{12}, \varphi_{12}\right), \mu_{T}\left(l_{\varphi_{12}}\right)\right) \\
& M_{\overline{\Upsilon_{T}}}\left(p_{12}\right)=\sup _{\varphi_{12} \in P} \min \left(S_{\omega_{12}}\left(p_{12}, \varphi_{12}\right), \mu_{T}\left(l_{\varphi_{12}}\right)\right) \\
& M_{\Upsilon_{T}^{\mathrm{C}}}\left(p_{12}\right)=\inf _{\varphi_{12} \in P} \max \left(\bar{S}_{\omega_{12}}\left(p_{12}, \varphi_{12}\right), \bar{\mu}_{T}\left(l_{\varphi_{12}}\right)\right) \\
& M_{\overline{\Upsilon_{T}^{\mathrm{C}}}}^{\overline{\bar{T}}}\left(p_{12}\right)=\sup _{\varphi_{12} \in P} \min \left(S_{\omega_{12}}\left(p_{12}, \varphi_{12}\right), \bar{\mu}_{T}\left(l_{\varphi_{12}}\right)\right)
\end{aligned}
$$

when tolerance indiscernibility relation is considered, with $\bar{S}_{\omega_{12}}\left(p_{12}, \varphi_{12}\right)=1-S_{\omega_{12}}\left(p_{12}, \varphi_{12}\right)$. In the aforementioned expressions, we use $\varphi_{12} \equiv\left\langle\varphi_{1}, \varphi_{2}\right\rangle$ and $\omega_{12} \equiv\left\langle\omega_{1}, \omega_{2}\right\rangle$, $\gamma$ denotes the number of granules formed in the universe $P$, and $m_{\digamma_{i} \omega_{12}}\left(p_{12}\right)$ gives the membership grade of $p_{12}$ in the $i$ th granule $\digamma_{i}^{\omega_{12}}$. These membership grades may be calculated using any concave, symmetric, and normal 2-D membership function (with support cardinality $\omega_{1} \times \omega_{2}$ ). Note that the sum of these membership grades over all the granules must be unity for a particular value of $p_{12}$. In (31), $S_{\omega_{12}}: P \times P \rightarrow$ $[0,1]$, which can be any concave and symmetric 2-D function, gives the relation between any two pixels in $P$. The value of $S_{\omega_{12}}\left(p_{12}, \varphi_{12}\right)$ is zero when the spatial separations between $p_{1}$ and $\varphi_{1}$, and $p_{2}$ and $\varphi_{2}$ are greater than $\omega_{1}$ and $\omega_{2}$, respectively, and $S_{\omega_{12}}\left(p_{12}, \varphi_{12}\right)$ equals unity when $p_{12}$ equals $\varphi_{12}$. The discussion in the case of grayness ambiguity measure, on the different forms that the lower and upper approximations of the sets $\Upsilon_{T}$ and $\Upsilon_{T}^{\complement}$ take, is also applicable here, when we consider $\digamma_{i}^{\omega_{12}}, S_{\omega_{12}}$, and $P$ instead of $\digamma_{i}^{\omega}, S_{\omega}$, and $G$, respectively.

We shall now quantify the ambiguities in the image $I$ by measuring the incompleteness of knowledge about the universe of pixels $P$. This measurement is done by calculating the following roughness values:

$$
\rho_{\omega}\left(\Upsilon_{T}\right)=1-\frac{\left|\Upsilon_{T}\right|}{\left|\overline{\Upsilon_{T}}\right|} \quad \rho_{\omega}\left(\Upsilon_{T}^{\mathcal{C}}\right)=1-\frac{\left|\underline{\mid \Upsilon_{T}^{\complement}}\right|}{\left|\overline{\Upsilon_{T}^{C}}\right|}
$$

where $\omega \equiv \omega_{12}$. Now, the ambiguity measure $\Lambda$ of $I$ is obtained as a function of $T$ as follows:

$$
\left.\Lambda_{\omega}^{L}(T)=-\frac{1}{2}\left[\varkappa\left(\Upsilon_{T}\right)\right)+\varkappa\left(\Upsilon_{T}^{\complement}\right)\right]
$$

where $\varkappa(D)=\rho_{\omega}(D) \log _{\beta}\left(\rho_{\omega}(D) / \beta\right)$ for any set $D \subseteq P$. Note that, in the aforementioned discussion, the ambiguity measure is obtained by using the roughness values associated with $\Upsilon_{T}$ and $\Upsilon_{T}^{\complement}$ in order to calculate the proposed logarithmic class of entropy measures that quantifies the incompleteness of knowledge about $P$. When the proposed exponential class of entropy measures is used, we get

$$
\Lambda_{\omega}^{E}(T)=\frac{1}{2}\left[\rho_{\omega}\left(\Upsilon_{T}\right) \beta^{\left(\bar{\rho}_{\omega}\left(\Upsilon_{T}\right)\right)}+\rho_{\omega}\left(\Upsilon_{T}^{\complement}\right) \beta^{\left(\bar{\rho}_{\omega}\left(\Upsilon_{T}^{\complement}\right)\right)}\right]
$$

where $\bar{\rho}_{\omega}(D)=1-\rho_{\omega}(D)$ for any set $D \subseteq P$.

The ambiguity measures obtained using (33) and (34) are referred to as the spatial ambiguity measures, and they lie in the range $[0,1]$, where a larger value means higher ambiguity.

\section{Average Image Ambiguity (AIA)}

As mentioned earlier, the elements in the set considered for quantifying ambiguities in a grayscale image hold a particular property, which is given by a membership function. This membership function is characterized by certain parameters, which, in turn, characterize the set under consideration. The property can be defined in different ways by changing some of these parameters. Different ambiguity $(\Lambda)$ measures are obtained for different definitions of the property, and the average of all these measures gives us a characteristic measure of the image under consideration. Therefore, we obtain a class of characteristic measures of an image based on rough set theory and its certain generalizations as follows:

$$
\widetilde{\Lambda}=\frac{1}{|\Theta|} \sum_{i \in \Theta} \Lambda(i)
$$

where $\Theta$ is a set of all possible combinations of values of the parameters that are used to define the property in different ways. We shall refer $\widetilde{\Lambda}$ as AIA, and its value lies in the range $[0,1]$, where a smaller value means that various parts of the image are better distinguishable from each other, in a holistic sense.

In our case, as mentioned earlier, we use the "darkness" property, and different definitions of this property can be obtained by changing a parameter $T \in G$, where $G$ is the universe of gray levels. Therefore, we get

$$
\begin{aligned}
& \widetilde{\Lambda_{\omega}^{L}}=\frac{1}{|G|} \sum_{T \in G} \Lambda_{\omega}^{L}(T) \\
& \widetilde{\Lambda_{\omega}^{E}}=\frac{1}{|G|} \sum_{T \in G} \Lambda_{\omega}^{E}(T)
\end{aligned}
$$


where $\widetilde{\Lambda_{\omega}^{L}}$ and $\widetilde{\Lambda_{\omega}^{E}}$ are the AIA measures obtained using the logarithmic and exponential classes of entropies, respectively. Note that $\omega$ in (36) is a constant with respect to the "darkness" property.

\section{APPLiCATIONS AND EXPERIMENTAL Results}

A plethora of image processing techniques based on measuring ambiguities in images using fuzzy set theory are available in literature, with some of them representing the state of the art. In this section, we shall demonstrate the utility of the proposed entropies in measuring ambiguities in images by considering a few elementary image processing applications, such as enhancement evaluation, segmentation, and edge detection, where ambiguity-measure-based techniques have been previously used.

As mentioned in Section I, ambiguities in images are due to fuzzy boundaries and rough resemblance. In Sections II and III, we have shown that the proposed classes of entropy measures based on rough set theory and its certain generalizations have the following advantages over most fuzzy-set-theory-based uncertainty measures.

1) There are no terms in the expressions of the proposed classes of entropy measure that "theoretically" convey the same.

2) Some of the proposed entropy measures can be used to quantify ambiguities due to both fuzzy boundaries and rough resemblance.

In this section, we shall also compare the use of the proposed entropies in measuring ambiguities with certain existing use of fuzziness measures in order to observe whether the aforementioned advantages translate into better performance. Thus, the effectiveness of some of the proposed entropy measures in quantifying ambiguities in images shall be demonstrated.

Throughout this section, we shall consider the proposed ambiguity measure given in (26), which signifies measuring the grayness ambiguity using the proposed logarithmic class of entropy functions. The measures in (25) which are used in (26) are calculated considering that the pairs of lower and upper approximations of the sets $\Upsilon_{T}$ and $\Upsilon_{T}^{C}$ represent a tolerance fuzzy rough-fuzzy set. The aforesaid statement, according to the terminology given in Section II-C, signifies that logarithmic tolerance fuzzy rough-fuzzy entropy is used in this section to get the grayness ambiguity measure. We consider the values of the parameters $\Delta$ and $\omega$ as eight and six gray levels, respectively, and the base $\beta$ as $e$, without loss of generality.

Note that the logarithmic tolerance fuzzy rough-fuzzy entropy is a representative of the proposed entropies which can be used to capture ambiguities due to both fuzzy boundaries and rough resemblance. The expression of this entropy measure, like those of all the other proposed entropy measures, does not have terms that "theoretically" convey the same. Hence, the utility of all the proposed entropy measures and the effectiveness of some of them can be demonstrated by considering the proposed logarithmic tolerance fuzzy rough-fuzzy entropy alone.

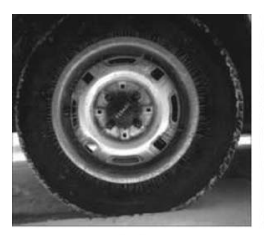

(a)

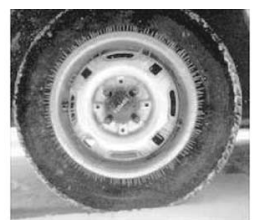

(b)

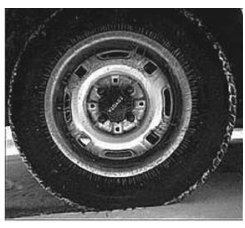

(c)
Fig. 5. Visual quality of the original and enhanced images of a tire (a) Original image. (b) Enhanced by histogram equalization. (c) Enhanced by unsharp masking.

\section{A. Enhancement Evaluation}

Quantitative evaluation of image enhancement operations is an important task in image processing. Among quite a few works of enhancement evaluation reported in literature, the one in [16] employs a fuzziness-based image quality measure. We shall now consider this image quality measure for enhancement evaluation and compare it to the use of the proposed logarithmic tolerance fuzzy rough-fuzzy entropy. If it is considered that the quality of an image is a term that describes how well its different parts are distinguishable, then the proposed AIA measures in (36) can readily be used as image quality measures for quantitative evaluation of image enhancement with a smaller value signifying better quality. Note that, on the contrary, a larger value of the measure used in [16] means better image quality.

Consider the images in Fig. 5. The image in Fig. 5(a) is the original image, and the images in Fig. 5(b) and (c) are the enhanced ones using the histogram equalization [17] and unsharp masking [17] techniques, respectively. The image quality measure used in [16] orders these images as (b), (a), and (c) with the measures $0.49309,0.3073$, and 0.27942 , respectively, whereas the AIA measure $\widetilde{\Lambda_{\omega}^{L}}$ orders these images as (c), (a), and (b) with the AIA values 0.20095, 0.22729, and 0.25046, respectively. The AIA values suggest that Fig. 5(c) has the best quality; as in Fig. 5(c), several details have cropped up due to the enhancement without compromising much on the overall contrast of the image, unlike Fig. 5(b).

\section{B. Segmentation}

Segmentation is one of the core tasks in image processing, and a vast number of simple to complex techniques have been reported in literature. In order to compare the use of the proposed logarithmic tolerance fuzzy rough-fuzzy entropy in segmentation with a fuzzy-set-theory-based method, we shall consider the fuzzy-entropy-based segmentation technique proposed in [18]. The technique in [18] uses a membership function like the one given in (20) and determines the fuzzy entropy measure of the underlying image for all values of $T$. The appropriate number of minima in the fuzzy entropy (as a function of $T$ ) curve is then chosen as the thresholds for segmentation. In order to have a fair comparison, we use the proposed ambiguity measure in (26) (based on the aforesaid entropy) instead of the fuzzy entropy in the same technique given in [18]. We apply the aforementioned segmentation algorithms to the grayscale images corresponding to Ohta's color features I1, I2 ${ }^{\prime}$, and I3 ${ }^{\prime}$ [19] of a color image and use a technique similar 


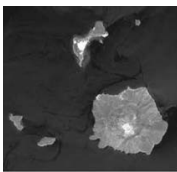

(a)

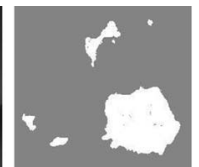

(b)

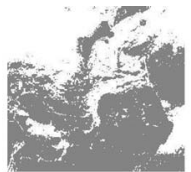

(c)

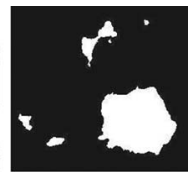

(d)
Fig. 6. Object extraction using algorithms employing the proposed grayness ambiguity measure and fuzzy entropy, and the mean shift method. (a) An image of islands. (b) Using proposed. (c) Using fuzzy entropy. (d) By mean shift method.

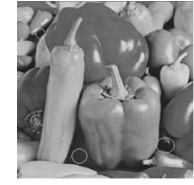

(a)

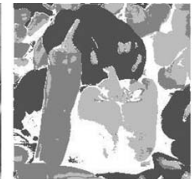

(b)

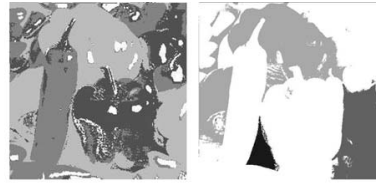

(d)
Fig. 7. Segmentation using algorithms employing the proposed grayness ambiguity measure and fuzzy entropy, and the mean shift method. (a) The pepper image. (b) Using proposed. (c) Using fuzzy entropy. (d) By mean shift method.

to the one used in [20] to obtain the segments in the color image. Secondary to visual inspection, we shall also use the $\beta$-index [21], where a larger value signifies better segmentation, to compare the performance of the algorithms.

We shall also consider a state-of-the-art segmentation technique proposed in [22], which uses the mean shift procedure, for comparison with the aforementioned segmentation technique based on the proposed ambiguity measure. The aforesaid comparison would let us know whether the segmentation results obtained by the proposed ambiguity-measure-based technique are comparable to that of the state-of-the-art technique, even when the technique using the proposed ambiguity measure considered here is not a sophisticated one. When color images are considered, the $\beta$-index cannot be used for the aforesaid comparison, as the mean shift segmentation method in [22] works on a color image as a whole, unlike the technique using the proposed ambiguity measure which works on the grayscale images corresponding to the underlying color features. It should be noted that the parameters required in the mean shift method (see [22]) are manually adjusted in accordance to the underlying segmentation problem.

In Fig. 6, the aforementioned algorithms are applied to separate the objects in the color image (intensity feature shown) from the backgrounds. The $\beta$-index values for the $\mathrm{I} 1, \mathrm{I} 2^{\prime}$, and $\mathrm{I} 3^{\prime}$ features of the image in Fig. 6(a) corresponding to the results in Fig. 6(b) and (c) are 7.162, 1.1985, and 1.9872 and 7.162, 2.6443 , and 1.8787 , respectively. It is visually evident that the algorithm using the proposed ambiguity measure outperforms the one using fuzzy entropy, and in this case, the larger $\beta$ index value for $\mathrm{I}^{\prime}$ corresponding to the result in Fig. 6(c) proves insignificant compared to the larger $\beta$-index value for I3' corresponding to the result in Fig. 6(b). As can be seen from Fig. 6(b) and (d), the object extraction results obtained by the algorithm using the proposed ambiguity measure are comparable to that of the mean shift method.

In Fig. 7, the aforementioned algorithms are applied to segment the color image (intensity feature shown) into specified

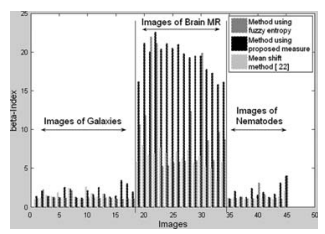

Fig. 8. Comparison of performance of the algorithms employing the proposed ambiguity measure and fuzzy entropy, and the mean shift method using the $\beta$-index measure.

numbers of regions. The $\beta$-index values for the $\mathrm{I} 1, \mathrm{I} 2^{\prime}$, and $\mathrm{I}^{\prime}$ features of the image in Fig. 7(a) corresponding to the results in Fig. 7(b) and (c) are 8.1027, 9.8338, and 28.399 and 7.4253, 9.9947, and 28.726, respectively. From visual inspection (the areas marked in circles) and the $\beta$-index values, we may say that the algorithm using the proposed measure outperforms the one using fuzzy entropy. Considering the comparison between the proposed ambiguity-measure-based algorithm and the mean shift method in Fig. 7, we find that there are considerable differences in the segmentation results obtained. These differences are due to the fact that the mean shift method forms regions in an image by considering a compromising combination of gray value/color similarity and spatial proximity, whereas the other two segmentation techniques mentioned in this section consider only the gray value/color similarity. It is visually evident in Fig. 7 that the algorithm using the proposed ambiguity measure gives better results than the mean shift method in terms of color uniformity within regions. On the other hand, the mean shift method outperforms the proposed ambiguity-measure-based algorithm when the compactness of a given region in terms of spatial proximity is considered.

In order to carry out a rigorous analysis, we first consider 45 grayscale images from the Universidad de Granada image database (http://decsai.ugr.es/cvg/dbimagenes/index.php), where images $1-18$ are that of galaxies, images 19-34 are Brain MRIs, and images 35-45 are that of nematodes. We then perform object/background separation in the images of galaxies and nematodes and segment the Brain MRIs into three regions employing the algorithms using the proposed ambiguity measure and fuzzy entropy, and the mean shift segmentation method. The corresponding $\beta$-index values are put against the image numbers in the bar chart shown in Fig. 8. It is evident from the figure that the algorithm using the proposed measure, in general, produces results which correspond to the larger $\beta$-index value signifying better segmentation performance than the algorithm using fuzzy entropy. Note that when a grayscale image is under consideration, the $\beta$-index evaluates segmentation performance in terms of gray value uniformity within regions. Therefore, as evident from Fig. 8, we find that the $\beta$-index suggests that the algorithm using the proposed ambiguity measure gives better results than the mean shift method, as the mean shift method compromises on the gray value similarity during segmentation.

\section{Edge Detection}

An important process in most of the edge detection systems existing in literature is to determine the edges through a 


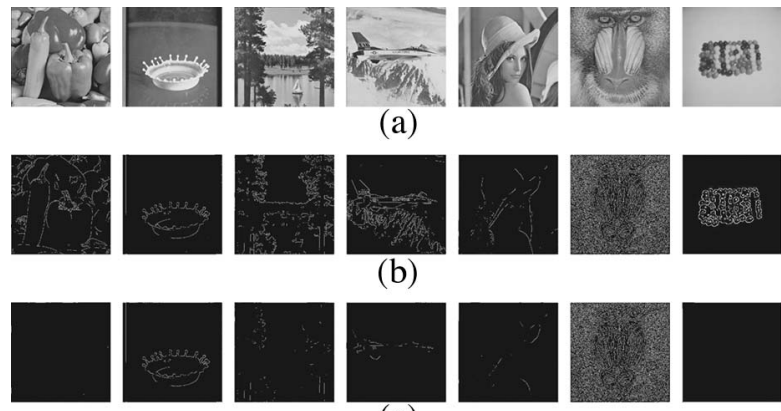

(c)

Fig. 9. Edge extraction using thresholds determined by algorithms employing the proposed ambiguity measure and fuzzy entropy. (a) The intensity features of the color images considered. (b) Extraction by algorithm using the proposed ambiguity measure. (c) Extraction by algorithm using fuzzy entropy.

decision making method after the edge strength at each pixel in the image has been obtained. A well-known example of such a decision making method is the hysteresis thresholding [23] which uses two predefined thresholds, where one of them is usually obtained by multiplying the other with a constant. The process of determining thresholds in histograms of edge strength, which are generally unimodal and positively (right) skewed, is considered here in order to compare the use of the proposed logarithmic tolerance fuzzy rough-fuzzy entropy with a fuzzy-set-theory-based method. We use the Canny operator for color images [24] and the nonmaximum suppression [23] to determine the edge strength at each pixel in a color image and then apply the previously mentioned algorithms, which use the proposed ambiguity measure and fuzzy entropy, in order to determine a threshold corresponding to each algorithm from the histogram of edge strength. Note that we have not used the threshold determined in the hysteresis process but instead used only the single threshold to extract the edges because our prime aim here is to compare the use of the proposed entropy with that of fuzzy entropy.

In Fig. 9, we consider a few color images such that the amount of edges present in them varies significantly. It is visually evident from the figure that the algorithm using the proposed measure satisfactorily extracts the edges in the images considered, whereas the algorithm using fuzzy entropy fails miserably in some. This shows that the ambiguities in images of edge strength are better represented by the proposed measure.

From the different image processing applications considered in this section, we see that quantifying ambiguities in images using the logarithmic tolerance fuzzy rough-fuzzy entropy, in general, results in better performance than the use of certain fuzziness measures like the fuzzy entropy. Note that, as mentioned earlier, the aforesaid proposed entropy used in this section is a representative of the proposed entropies which can be used to capture ambiguities due to both fuzzy boundaries and rough resemblance. Thus, the effectiveness of some of the proposed entropies, which can be used to capture ambiguities due to both fuzzy boundaries and rough resemblance in images, is demonstrated by the performance improvement observed. We have also carried out analyses considering the quantification of spatial ambiguity, where similar performance improvement has been observed.
Note that, as mentioned earlier, we have taken specific values of the parameters $\Delta, \omega$, and $\beta$ in this section to calculate grayness ambiguity measure. In order to calculate spatial ambiguity measure, apart from $\Delta$ and $\beta$, we need to assign values of the parameters $\omega_{1}$ and $\omega_{2}$ instead of $\omega$. Assigning appropriate values to the aforesaid parameters is a matter of subjective analysis. As analyzed in [25], in order to get a suitable value of $\Delta$, the global gray value distribution of the underlying image can be considered. The width of base regions corresponding to all the peaks in the distribution may then be found, and as mentioned in [25], certain base regions with widths below a certain threshold may be marked as "unnecessary". The minimum of half of the width of the remaining base regions can be chosen as the value of $\Delta$. The value of $\omega$ can be based on Weber's law [26] and the related concept of just noticeable difference. Following Weber's law, the difference of any gray value from another that makes them just distinguishable can be considered as the value of $\omega$. Choosing appropriate values of $\omega_{1}$ and $\omega_{2}$ is analogous to the problem of choosing a suitable window size, which is encountered in many image processing tasks. In most of such image processing tasks, the window size is chosen arbitrarily as $3 \times 3$ or $5 \times 5$, and in similar manner, one can consider that both $\omega_{1}$ and $\omega_{2}$ equal three or five. A difference in the choice of the base $\beta$ amounts only to a change in the unit of measuring ambiguity. Therefore, any suitable value of $\beta$ can be considered, and the value $\beta$ must not be changed through an experiment.

\section{CONCLUSION}

Ambiguities in grayscale images are due to fuzzy boundaries between regions, and rough resemblance between nearby gray levels and between values at nearby pixels. The use of rough set theory and its certain generalizations for quantifying ambiguities in images has been proposed in this paper. New classes of entropy measures based on rough set theory and its certain generalizations have been proposed, and rigorous theoretical analysis of the proposed entropies has been carried out. The proposed entropies have then been used to quantify ambiguities in images, and it has been shown that some of the proposed entropies can be used to quantify ambiguities due to both fuzzy boundaries and rough resemblance. The utility and effectiveness of the proposed entropy measures have been demonstrated by considering some elementary image processing applications and comparisons with the use of certain fuzziness measures. A new measure called average image ambiguity has also been defined in this context.

The proposed classes of entropy measures based on rough set theory and its certain generalizations are not restricted to the few applications discussed in this paper. They are, in general, applicable to all tasks where ambiguity-measure-based techniques have been found suitable, provided that the rough resemblance aspect of ambiguities exists. It would be interesting to carry out such investigations as the proposed measures possess certain advantages over most fuzzy-set-theory-based uncertainty measures, which have been the prime tool for measuring ambiguities. 


\section{ACKNOWLEDGMENT}

The authors would like to thank the anonymous referees for their valuable suggestions. S. K. Pal would also like to thank the Government of India for the J. C. Bose National Fellowship.

\section{REFERENCES}

[1] G. Klir and B. Yuan, Fuzzy Sets and Fuzzy Logic: Theory and Applications. New Delhi, India: Prentice-Hall, 2005.

[2] S. K. Pal, "Fuzzy models for image processing and applications," Proc. Indian Nat. Sci. Acad., vol. 65, no. 1, pp. 73-90, 1999.

[3] Z. Pawlak, Rough Sets: Theoretical Aspects of Reasoning About Data. Dordrecht, The Netherlands: Kluwer, 1991.

[4] D. Dubois and H. Prade, "Rough fuzzy sets and fuzzy rough sets," Int. J. Gen. Syst., vol. 17, no. 2/3, pp. 191-209, 1990.

[5] A. Skowron and J. Stepaniuk, "Tolerance approximation spaces," Fundamenta Informaticae, vol. 27, no. 2/3, pp. 245-253, Aug. 1996.

[6] T. Beaubouef, F. E. Petry, and G. Arora, "Information-theoretic measures of uncertainty for rough sets and rough relational data bases," Inf. Sci., vol. 109, no. 1-4, pp. 185-195, Aug. 1998.

[7] M. J. Wierman, "Measuring uncertainty in rough set theory," Int. J. Gen. Syst., vol. 28, no. 4, pp. 283-297, 1999.

[8] J. Liang, K. S. Chin, C. Dang, and R. C. M. Yam, "A new method for measuring uncertainty and fuzziness in rough set theory," Int. J. Gen. Syst., vol. 31, no. 4, pp. 331-342, 2002.

[9] J.-S. Mi, X.-M. Li, H.-Y. Zhao, and T. Feng, "Information-theoretic measure of uncertainty in generalized fuzzy rough sets," in Rough Sets, Fuzzy Sets, Data Mining and Granular Computing. Berlin, Germany: Springer-Verlag, 2007, pp. 63-70.

[10] R. R. Yager, "Entropy measures under similarity relations," Int. J. Gen. Syst., vol. 20, no. 4, pp. 341-358, 1992.

[11] S. K. Pal, B. U. Shankar, and P. Mitra, "Granular computing, rough entropy and object extraction," Pattern Recognit. Lett., vol. 26, no. 16, pp. 2509-2517, Dec. 2005.

[12] D. Sen and S. K. Pal, "Histogram thresholding using beam theory and ambiguity measures," Fundamenta Informaticae, vol. 75 , no. 1-4, pp. 483-504, Jan. 2007.

[13] N. R. Pal and S. K. Pal, "Entropy: A new definition and its applications," IEEE Trans. Syst., Man, Cybern., vol. 21, no. 5, pp. 1260-1270, Sep./Oct. 1991.

[14] N. R. Pal and J. C. Bezdek, "Measuring fuzzy uncertainty," IEEE Trans. Fuzzy Syst., vol. 2, no. 2, pp. 107-118, May 1994.

[15] B. R. Ebanks, "On measures of fuzziness and their representations," J. Math. Anal. Appl., vol. 94, no. 1, pp. 24-37, Aug. 1983.

[16] H. R. Tizhoosh, G. Krell, and B. Michaelis, " $\lambda$ enhancement: Contrast adaptation based on optimization of image fuzziness," in Proc. IEEE Int. Conf. Fuzzy Syst., 1998, vol. 2, pp. 1548-1553.

[17] R. C. Gonzalez and R. E. Woods, Digital Image Processing, 2nd ed. New Delhi, India: Pearson Educ., 2002.

[18] S. K. Pal, R. A. King, and A. A. Hashim, "Automatic grey level thresholding through index of fuzziness and entropy," Pattern Recognit. Lett., vol. 1, no. 3, pp. 141-146, 1983.

[19] Y. I. Ohta, T. Kanade, and T. Sakai, "Color information for region segmentation," Comput. Graphics Image Process., vol. 13, no. 3, pp. 222241, Jul. 1980.

[20] Y. W. Lim and S. U. Lee, "On the color image segmentation algorithm based on the thresholding and the fuzzy C-means techniques," Pattern Recognit., vol. 23, no. 9, pp. 935-952, 1990.

[21] S. K. Pal, A. Ghosh, and B. U. Shankar, "Segmentation of remotely sensed images with fuzzy thresholding, and quantitative evaluation," Int. J. Remote Sens., vol. 21, no. 11, pp. 2269-2300, Jul. 2000.

[22] D. Comaniciu and P. Meer, "Mean shift: A robust approach toward feature space analysis," IEEE Trans. Pattern Anal. Mach. Intell., vol. 24, no. 5, pp. 603-619, May 2002.

[23] J. Canny, "A computational approach to edge detection," IEEE Trans. Pattern Anal. Mach. Intell., vol. PAMI-8, no. 6, pp. 679-698, Nov. 1986

[24] A. Koschan and M. Abidi, "Detection and classification of edges in color images," IEEE Signal Process. Mag., vol. 22, no. 1, pp. 64-73, Jan. 2005.

[25] C. A. Murthy and S. K. Pal, "Histogram thresholding by minimizing graylevel fuzziness," Inf. Sci., vol. 60, no. 1/2, pp. 107-135, Mar. 1992.

[26] A. K. Jain, Fundamentals of Digital Image Processing. New Delhi, India: Prentice-Hall, 2001.

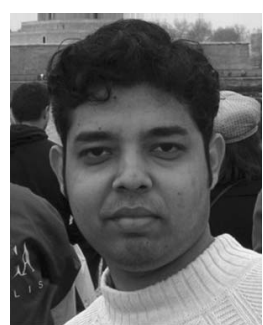

Debashis Sen (S'05-M'06) received the M.A.Sc. degree in electrical engineering from Concordia University, Montreal, QC, Canada, in 2005 and the B.Eng. (Hons.) degree in electronics and communication engineering from the University of Madras, Chennai, India, in 2002. He is currently working toward the Ph.D. degree in computer engineering at the Center for Soft Computing Research, Indian Statistical Institute, Calcutta, India.

He was with the Center for Signal Processing and Communications and with the Video Processing and Communications Group, Concordia University, in 2003-2005. His research interests include image and video processing, probability theory, and soft computing.

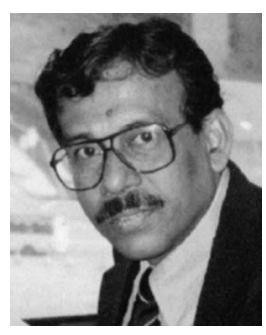

Sankar K. Pal (M'81-SM'84-F'93) received the $\mathrm{Ph} . \mathrm{D}$. degree in radio physics and electronics from the University of Calcutta, West Bengal, India, in 1979 and another $\mathrm{Ph} . \mathrm{D}$. degree in electrical engineering along with DIC from Imperial College, University of London, London, U.K., in 1982.

He is the Director and a Distinguished Scientist of the Indian Statistical Institute. Currently, he is also a J. C. Bose Fellow of the Government of India. $\mathrm{He}$ founded the Machine Intelligence Unit and the Center for Soft Computing Research: A National Facility in the Institute in Calcutta. He was with the University of California, Berkeley, and the University of Maryland, College Park, in 1986-1987; the NASA Johnson Space Center, Houston, TX, in 1990-1992 and 1994; and the U.S. Naval Research Laboratory, Washington, DC, in 2004.

Dr. Pal is a Fellow of the Academy of Sciences for the Developing World (TWAS), Italy, the International Association for Pattern Recognition, U.S., the International Association of Fuzzy Systems, U.S., and all the four National Academies for Science/Engineering in India. Since 1997, he has been a Distinguished Visitor of the IEEE Computer Society (U.S.) for the Asia-Pacific Region and has been holding several visiting positions in Hong Kong and Australian universities. He is a coauthor of 14 books and more than 300 research publications in the areas of pattern recognition and machine learning, image processing, data mining and Web intelligence, soft computing, neural nets, genetic algorithms, fuzzy sets, rough sets, and bioinformatics. He has received the 1990 S.S. Bhatnagar Prize (which is the most coveted award for a scientist in India) and many prestigious awards in India and abroad, including the 1999 G.D. Birla Award, the 1998 Om Bhasin Award, the 1993 Jawaharlal Nehru Fellowship, the 2000 Khwarizmi International Award from the Islamic Republic of Iran, the 2000-2001 FICCI Award, the 1993 Vikram Sarabhai Research Award, the 1993 NASA Tech Brief Award (U.S.), the 1994 IEEE Transactions on Neural Networks Outstanding Paper Award (U.S.), the 1995 NASA Patent Application Award (U.S.), the 1997 IETE-R.L. Wadhwa Gold Medal, the 2001 INSA-S.H. Zaheer Medal, the 2005-2006 ISC-P.C. Mahalanobis Birth Centenary Award (Gold Medal) for Lifetime Achievement, the 2007 J. C. Bose Fellowship of the Government of India, and the 2008 Vigyan Ratna Award from Science and Culture Organization, West Bengal. Prof. Pal is/was an Associate Editor for the IEEE TRANSACTIONS ON PATTERN ANALYSIS AND MACHINE INTELLIGENCE (2002-2006), the IEEE Transactions ON NeURAL Networks [1994-1998 and 2003-2006], Neurocomputing (1995-2005), Pattern Recognition Letters, the International Journal of Pattern Recognition and Artificial Intelligence, Applied Intelligence, Information Sciences, Fuzzy Sets and Systems, Fundamenta Informaticae, the LNCS Transactions on Rough Sets, the International Journal of Computational Intelligence and Applications, IET Image Processing, the Journal of Intelligent Information Systems, and the Proceedings of INSA-A. He is also a Book Series Editor of Frontiers in Artificial Intelligence and Applications (IOS Press) and Statistical Science and Interdisciplinary Research (World Scientific, Singapore); a member of the Executive Advisory Editorial Board of the IEEE TRANSACTIONS ON FUZZY SYSTEMS, the International Journal of Image and Graphics, and the International Journal of Approximate Reasoning; and a Guest Editor for the IEEE Computer. 